\title{
Search for kaonic bound states
}

\section{Robert Muenzer*i}

Technische Universität Muenchen

E-mail: rmuenzer@ph.tum.de

\begin{abstract}
The investigation of the kaon-nucleon interaction has been intensified in the last year due to new results on $\Lambda(1405)$ [1] and indications on the existence of the $p p K^{-}$bound state [2].

In a dedicated experiment at the FOPI Sepctrometer at GSI in Darmstadt the possible production of this state in proton proton collision is investigated. During data taking an additional detector was used to enhance events containing Lambda Hyperons.

This contribution will show the status of the ongoing analysis, expecially the reconstuction of the $\mathrm{p} K^{+} \Lambda$ final state, with the help of a kinemtical refit tool, which allows an improvement of the signal quality.
\end{abstract}

51st International Winter Meeting on Nuclear Physics

21-25 January 2013

Bormio (Italy)

\footnotetext{
${ }^{*}$ Speaker.

${ }^{\dagger}$ This work was sponsored by the TUM Graduate School
} 


\section{Introduction}

\subsection{Motivation}

The investigation of the kaon-nucleon interaction has been intensified in the last years due to new results on $\Lambda(1405)$ and indications on the existence of the $p p K^{-}$bound state [1]. The possible creation of the $p p K^{-}$has been investigated with the FOPI spectrometer at GSI in proton-protoncollisions at $3.1 \mathrm{GeV}$ beam kinetic energy. Indeed, according to some theoretical predictions, this reaction should favor the formation of the $p p K^{-}$[2].

\subsection{The FOPI spectrometer at GSI}

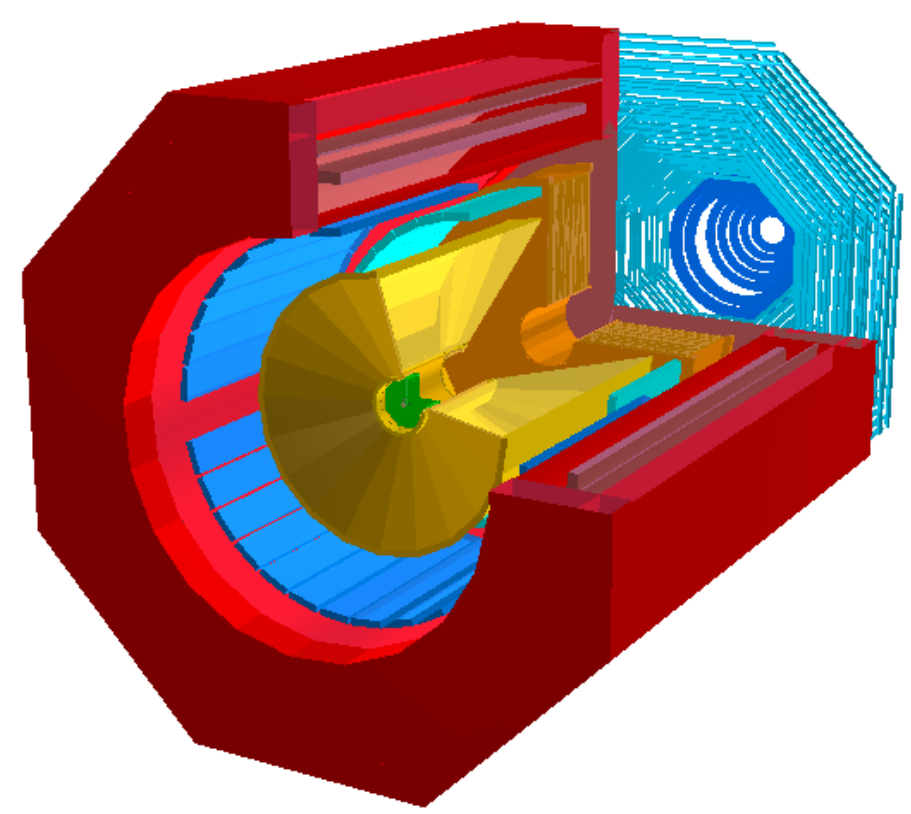

Figure 1: Schematic picture of the FOPI Spectrometer. The drift chamber CDC (yellow) and Helitron (orange) are located inside the solenoid magnet (red). The CDC is surrounded by the barrel scintillator (blue) and the RPC barrel (turquoise). Behind the magnet is the time-of-flight wall out of plastic scintillators (turquoise)

For the exclusive measurements of the $p p K^{-}$a detector with a high geometrical acceptance at a facility, which is able to produce a proton beam up to $3.5 \mathrm{GeV}$ is necessary. These conditions are fulfilled at the FOPI Spectrometer at the SIS18 at GSI in Darmstadt [5]. This spectrometer has an geometrical acceptance from $5^{\circ}$ to $113^{\circ}$ in the polar angle and nearly $360^{\circ}$ in the azimuthal angle. The FOPI spectrometer is shown on fig. 1. It consists of two drift chambers, the Helitron for lower and the CDC for higher polar angles (yellow). The CDC is surrounded by a scintillator(blue) and a RPC (turquoise) time of flight barrel. All these detector are located inside a 0.6 Tesla solenoid magnetic field. The PLAWA, which measures the time of flight at small polar angles, completes the FOPI spectrometer.

The track reconstruction happens in two different detector systems, which cover separate hemispheres. The track in the forward hemisphere are reconstructed via the Helitron and afterwards 
combined with the hit position and flight time information of corresponding hit in the Plastic Wall. To avoid ghost tracks only the candidates with a valid hit in the Plastic Wall are taken into account. The backward hemisphere tracks are reconstructed by the Central Drift Chamber. For a better particle identification those tracks can be combined with hit point in the RPC barrel, which is necessary for identification of $K^{+}$(see 3.1) .

\section{The $\Lambda$ trigger}

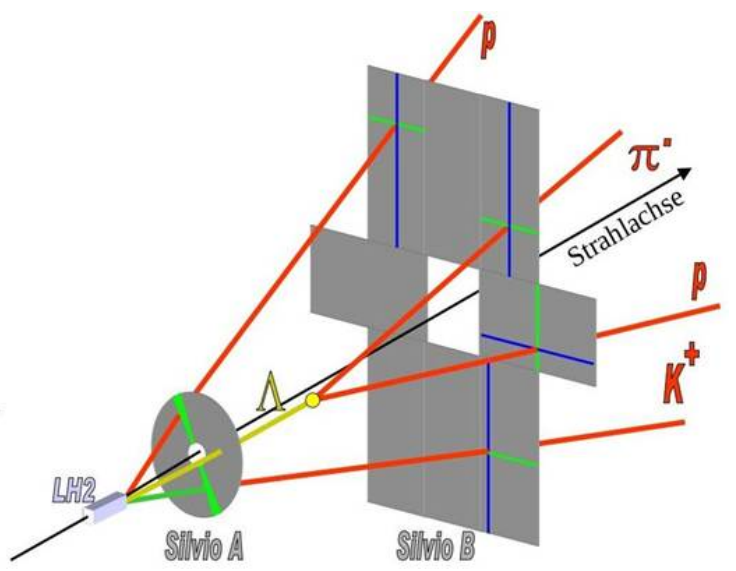

Figure 2: Schematic view of the two layers of $\mathrm{Si} \Lambda \mathrm{ViO}$ detector with a typical reaction $\mathrm{p}+\mathrm{p}->\mathrm{p}+K^{+}+\Lambda$. Only the charged particle $K^{+}$and p are inducing a signal in the first layer. Due to the decay of the $\Lambda$ in $p$ and $\pi^{-}$the number of inducted hits is higher in the second layer.

Additionally to the FOPI spectrometer a silicon detector system has been constructed and was placed close to the target. This detector was build for two purposes. First it was built to enhance to amount of events containing a $\Lambda$ particles. The detector consists of two layers of silicon detectors, which distances to the target are arranged in such a way, that around $60 \%$ of the $\Lambda$ particle $(c \tau=7,89 \mathrm{~cm})$ are decaying between both layers. Fig. 2 shows a schematic view of the detector and its working principle for a typical reaction $p+p \rightarrow p+K^{+}+\Lambda$. The neutral particle does not induce a signal in the first layer and decays between the two layers, therefore the number of particle hits is higher in the second, than in the first layer. This property is used to create a trigger signal, which enhances the amount of events containing a $\Lambda$ particle [3] in the data. With this trigger condition about $70 \cdot 10^{6}$ events have been collected.

Furthermore the hit point information of the second detector is matched with the track information of the forward hemisphere tracks. Due to this additional track point near the target it is possible to improve the momentum resolution of about $10 \%$ [3].

\section{Analysis steps}

From the recorded events, the exclusive production of the final state of $p+K^{+}+\Lambda\left[\rightarrow p+\pi^{-}\right]$ has to be extracted. In a first step events which contain three positive and one negative charged tracks are collected. In the second only those events are selected which contain a $K^{+}$candidate. 
The selection is described in 3.1 .

In a third step $\Lambda$ particles are identified via their decay in $p+\pi^{-}$(3.2). In the last step the exclusive reactions are selected by energy and momentum conservation constraints.

\subsection{Kaon selection}

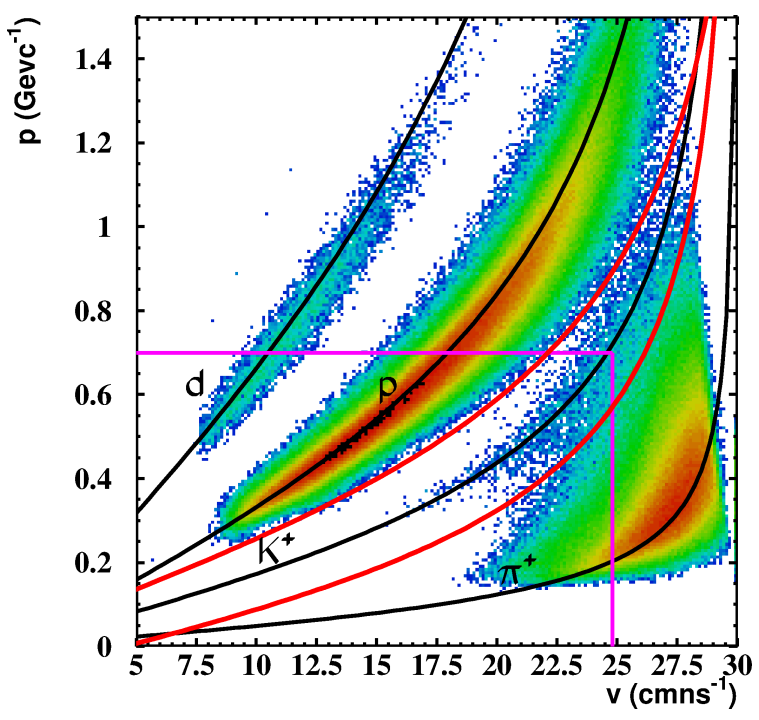

Figure 3: Momentum versus velocity of CDC-RPC tracks. Black lines show the distribution for the nominal particle masses. Pink lines show the border of clear $K^{+}-\pi^{+}$separation. $K^{+}$Candidates are selected between the red lines.

Kaon Candidates are selected in the backward hemisphere in the angular region of the RPC Barrel detectors, since a valid velocity information of the particle is necessary in combination with the reconstructed momentum in the central drift chamber. Fig. 3 shows the particle momentum versus velocity. Different particle species are indicated by their nominal $\beta \gamma$-curves in black lines. Non strange particles like pions and protons are clearly separable. The separation of $K^{+}$from protons is quite good over the whole momentum range, for $\pi^{+}$a clear separation is possible up to a momentum of $0,6 \mathrm{GeVc}^{-1}$ and a velocity of $28 \mathrm{cmns}^{-1}$ (fig. 3 violett lines).

$\mathrm{K}^{+}$candidates are selected with the red band which is visible in fig. 3. Since in the further analysis exclusive reactions will be identified, kaon candidates with a higher velocity than $28 \mathrm{cmns}^{-1}$ can be selected, even the separation would not be sufficient for an inclusive analysis.

\section{2 $\Lambda$ reconstruction}

In a next step $\Lambda$ particles are reconstructed via the decay into $p-\pi^{-}$-pairs. In fig. 6 (a) the invariant mass distribution of $\mathrm{p}-\pi^{-}$pairs is shown by the black crosses. The background is obtained by a simultanious fit of an polynomial and a gaussian function. The resulting background distribution is shown by the red line.

Fig. 6 (b) shows the background subtracted signal. The resulting $\Lambda$-particle have a centroid mass of $m_{\Lambda}=1,113 \mathrm{GeVc}^{-2}$ and a width $\sigma_{\Lambda}=10,7 \mathrm{MeVc}^{-2}$. 

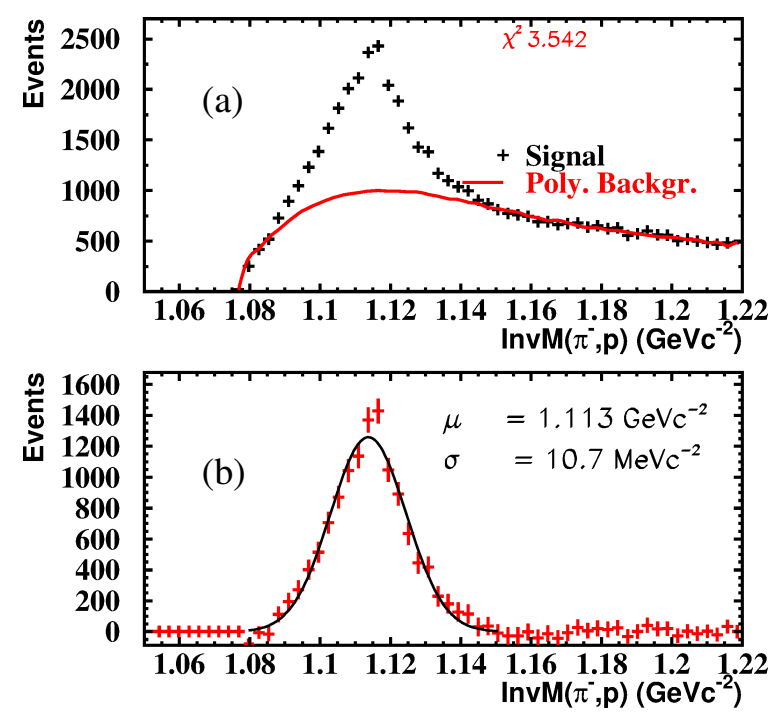

Figure 4: Invariant mass of $p-\pi^{-}$-pairs after the $K^{+}$selection.

\subsection{Exclusive selection via kinematical refit}

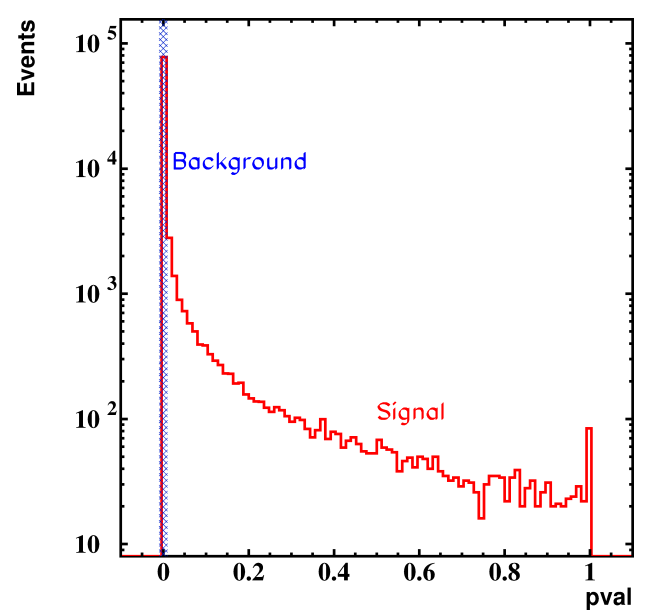

Figure 5: Confidence Level (P-val) distribution of the kinematical refit with energy and momentum conservation. The blue shaded are indicates background events.

The final step to identify the reaction $p+p \rightarrow p+K^{+}+\Lambda$ is the selection of exclusive reactions. This is done by asking for energy and momentum conservation.

Since the particles are identified in different hemispheres with different correlated errors for the track parameters, a procedure has to be used, which takes the different errors into account. That procedure - named kinematical refit (3.3) - varies the four-momenta of all particle that have been associated to the exclusive reaction within their errors until the constraint of energy and momentum conservation is fulfilled([4]). After the refit procedure for each particle new four-momentum is available, which fulfill the applied constraint. The confidence level (p-value) of the kinematical refit can be used to separate signal from background events. Values close to zero are background 
events, which do not fulfil exclusivity within the track parameter errors. By cutting on the confidence level the signal events can be extracted. The cut value was obtained by the maximization of the Signal $^{2} /$ Background value.Fig. 5 shows the p-value distribution. Fig. 6 shows the $\Lambda$ spectrum
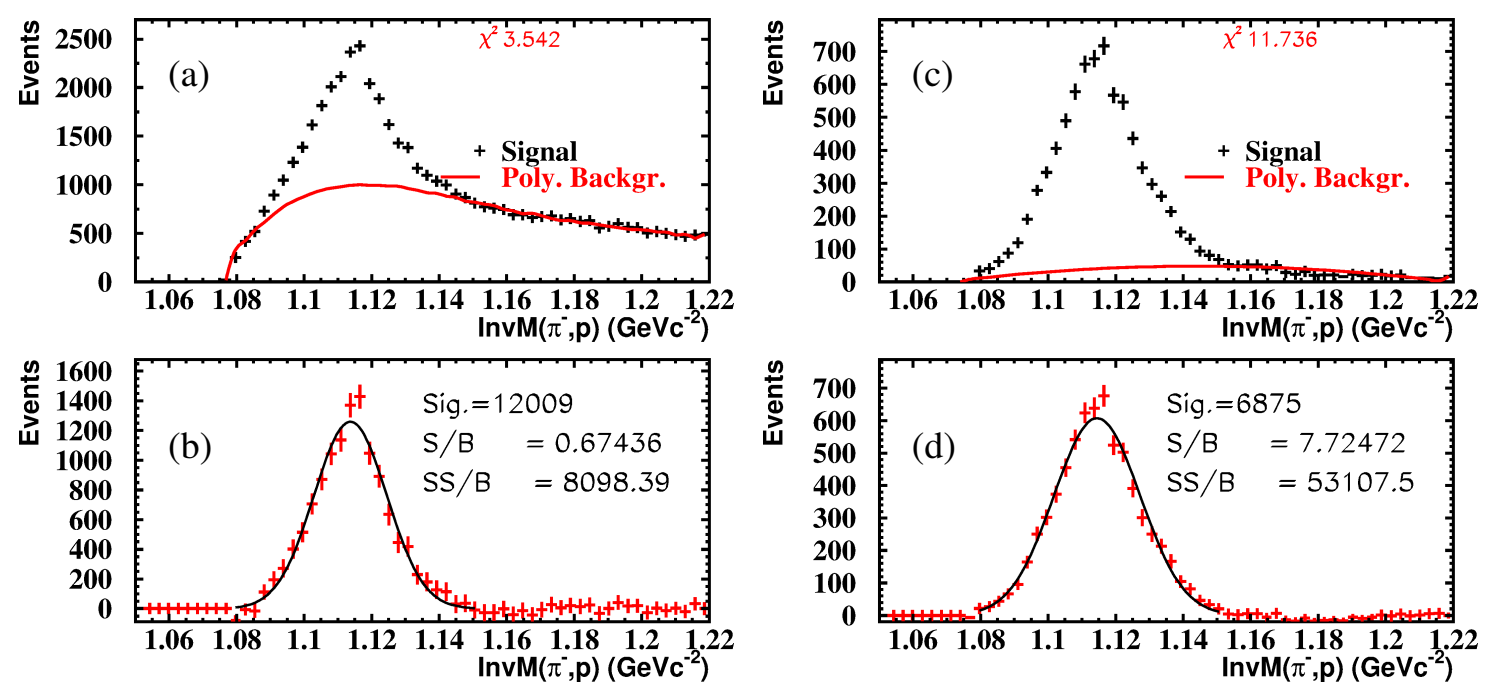

Figure 6: Invariant mass of $\mathrm{p}$ and $\pi^{-}$after $K^{+}$candidates selection (a). After exclusivity selection and background selection by kinematical refit.

before the cut on the refit quality (a) and after that cut (b). One can clearly see, that the signal to background increases from 0.67 to 7.7, while the total signal gets from around 12000 to 6800 .

This increase of the signal to background ratio by a factor 11 shows that the kinematical refit is a very nice tool to suppress background origintating originate from non exclusive events.

\section{Summary and outlook}

For the proton-proton experiment we installed a new detector inside the FOPI spectrometer. This detector allows to enhance the amount of $\Lambda$ containing events which is the base for the investigation the possible existance of the $p p K^{-}$.

After the selection of $K^{+}$with the help of RPC we suppress the non exclusive background quite sufficient and extract around 6800 Events with the final state of $p+\Lambda+K^{+}$.

Further steps the contribution of the reaction $p+p \rightarrow p+K^{+}+\Sigma[\rightarrow \Lambda+\gamma]$ will be analysed.

This channel has the final state like the one, we are interested in and differs only in the production of an additional $\gamma$, which could not be detetcted by our spectrometer.

After the suppresision of this contribution, a thorough analysis of the final state $p K^{+} \Lambda$ with the help of partcile waves analysis techniques is planned.

\section{References}

[1] L. Fabbietti, J. Siebenson / arXiv:1208.0205

[2] T. Yamazaki, M. Maggiora, P. Kienle / PRL 104 / 132502 (2010) 
[3] Si $\Lambda$ ViO - A Trigger for $\Lambda$-Hyperons - in print

[4] Diploma Thesis Dominik Pleiner https ://www.e12.ph.tum.de/groups/kcluster/Documents/Publications/Thesispleiner.pdf

[5] J.Ritman and others,Nucl.Phys.Proc.Suppl.44,1995,708-715 